\section{CPS-177 DEALING WITH IATROGENIC CARDIAC ARREST IN PSYCHIATRY, DO NOT OVERLOOK MONITORING!}

${ }^{1} \mathrm{~S}$ Wise, ${ }^{1} \mathrm{C}$ Kowal ${ }^{*},{ }^{2} \mathrm{~B}$ Pignon, ${ }^{3} \mathrm{~K}$ Richard, ${ }^{2} \mathrm{~S}$ Vanhulst, ${ }^{2} \mathrm{~A}$ Pelissolo, ${ }^{1} \mathrm{M}$ Dalle-Pecal, ${ }^{4} \mathrm{M}$ Paul, ${ }^{1} \mathrm{C}$ Divine. ${ }^{1}$ Albert Chenevier Hospital - Henri Mondor Hospital Group - AP-HP, Pharmacy Department, Creteil, France; ${ }^{2}$ Albert Chenevier Hospital - Henri Mondor Hospital Group - AP-HP, Psychiatric Department, Creteil, France; ${ }^{3}$ Albert Chenevier Hospital - Henri Mondor Hospital Group - AP-HP, Cardiac Rehabilitation Department, Creteil, France; ${ }^{4}$ Henri Mondor Hospital - Henri Mondor Hospital Group - AP-HP, Pharmacy Department, Creteil, France

\subsection{6/ejhpharm-2019-eahpconf.326}

Background In 2017 a patient's death occurred in the psychiatry department of our establishment. After a morbidity-mortality review, the hypothesis of a cardiac arrest after intake of torsadogenic drugs has been suggested.

Purpose The state of cardiac patient care in our psychiatry units was one of the strategic axes retained to define priority actions for improvement.

Material and methods Records of the hospitalised psychiatry patients were analysed on a given day in April 2018. A literature review allowed selection of the factors to analyse: ionogram dates and results, thyroid function, arterial tension (AT), heart rhythm (HR), electrocardiogram realisation, corrected QT interval (QTc), torsadogenic risk factors (female $\geq 65$ years, ischaemic heart disease, torsadogenic drug) and co-prescriptions of psychotropic drugs inducing QT prolongation (PDIQTP). Only the factors traced in the patients' records during the first 30 days of hospitalisation were analysed.

Results Ninety-six records were analysed $(100 \%$ of inpatients). Found at admission were ionograms, thyroid function, AT, HR and electrocardiogram realisation, respectively for 94\%, 70\%, 95\%, 96\% and 90\% of patients. Seven hypokalaemias were found and were all adjusted during the first month. No hypocalcaemia or hypothyroidism were found but one hyperthyroidism was revealed and explored. Seven hypertensions were explored. No bradycardia was recorded. Four patients had QTc prolongation $(\geq 450 \mathrm{~ms})$. Among them, two profited from an additional electrocardiogram. The percentage of patients with one risk factor was 19\% and $2 \%$ of patients had more than one risk factor. Half of these patients underwent an additional electrocardiogram. During hospitalisation, 44 PDIQTP, 17 initiations and 12 raises of torsadogenic drug dosage were carried out. These modifications were monitored by an extra electrocardiogram in $13 \%$ of cases.

Conclusion Admission cardiac check-up was mainly realised and its disturbances corrected or explored. However, the thyroid function was underestimated whereas its disturbance can cause not only cardiac disorders but also psychiatric disorders. Furthermore, in risk situations that need an extra electrocardiogram during hospitalisation (QTc prolongation for example), cardiac monitoring was insufficient. These two points will be spotlighted in a cardiac monitoring protocol for psychiatry inpatients, in order to prevent iatrogenic cardiac arrests throughout the hospitalisation.

\section{REFERENCES AND/OR ACKNOWLEDGEMENTS}

No conflict of interest.
4CPS-178 ABSTRACT WITHDRAWN 